\title{
A Nonlinear Implicit Fractional Equation with Caputo Derivative
}

\author{
Ameth Ndiaye \\ Département de Mathematiques, FASTEF, UCAD, Dakar, Senegal \\ Correspondence should be addressed to Ameth Ndiaye; ameth1.ndiaye@ucad.edu.sn \\ Received 21 January 2021; Revised 10 February 2021; Accepted 19 February 2021; Published 5 March 2021 \\ Academic Editor: Zakia Hammouch \\ Copyright (c) 2021 Ameth Ndiaye. This is an open access article distributed under the Creative Commons Attribution License, \\ which permits unrestricted use, distribution, and reproduction in any medium, provided the original work is properly cited. \\ In this paper, we study a nonlinear implicit differential equation with initial conditions. The considered problem involves the \\ fractional Caputo derivatives under some conditions on the order. We prove an existence and uniqueness analytic result by \\ application of Banach principle. Then, another result that deals with the existence of at least one solution is delivered and some \\ sufficient conditions for this result are established by means of the fixed point theorem of Schaefer. At the end, we discuss two \\ examples to illustrate the applicability of the main results.
}

\section{Introduction}

The theory of differential equations of fractional order and fractional calculus is very important since they can be used in analyzing and modeling real word phenomena. Recently, several researchers are interested in the important progress of differential equations of fractional order. For more information on these works and their applications, one can consult the references [1-9]. In particular, research on the existence of unique solutions for fractional differential equations is of big importance since it helps physicians to better understand the behaviour of real phenomena. See, for more details, the references [10-14].

The motivation for this work arises from both the development of the theory of fractional calculus itself and its wide applications to various fields of science, such as physics, chemistry, biology, electromagnetism of complex media, robotics, and economics.
Much attention has been paid to the existence and uniqueness of solutions of fractional dynamical systems [15-18] due to the fact that existence is the fundamental problem and a necessary condition for considering some other properties for fractional dynamical systems, such as controllability and stability. Chai [19] provided sufficient conditions for the existence of solutions to a class of antiperiodic boundary value problems for fractional differential equations, while Sheng and Jiang [20] considered a class of initial value problems for fractional differential systems. There are several operators studied in the field of fractional calculus, for example, see [21-26], but the difference in this work is that the operator considered is in the sense of Caputo derivative.

Motivated by the works of Benchohra et al. [27], we will establish in this paper existence and uniqueness results of the solutions of the fractional dynamical system with Caputo fractional derivative 


$$
\left\{\begin{array}{l}
D^{\alpha} x(t)-A D^{\beta} x(t)=f\left(t, x(t), D^{\beta} x(t), D^{\alpha} x(t)\right), \quad t \in I=[0,1] \\
x(0)=x_{0} \\
x^{\prime}(0)=x_{0}^{\prime} \\
x^{\prime \prime}(0)=x_{0}^{\prime \prime} \\
x^{\prime \prime \prime}(0)=x_{0}^{\prime \prime}
\end{array}\right.
$$

where $D^{\alpha}$ is in the sense of Caputo, $f: I \times \mathbb{R}^{n} \times \mathbb{R}^{n} \longrightarrow \mathbb{R}^{n}$ is a given function, $x_{0}, x_{0}^{\prime}, x_{0}^{\prime \prime} \in \mathbb{R}^{n}, A$ is an $n \times n$ matrix, and $1<\beta<2,3<\alpha<4$, with $\beta+2<\alpha$.

Rest of the paper is organised as follows: in Section 2, we recall some results and definitions which we use for the proof of our main results. In Section 3, we give and prove the main theorems of this paper, and we discuss some illustrative examples.

\section{Preliminaries}

In this section, we introduce some definitions, lemmas, and preliminaries facts which are used throughout this paper. See [7] for more information. Let |.| be a suitable norm in $\mathbb{R}^{n}$ and $\|$.$\| be the matrix norm. We denote by C\left(I, \mathbb{R}^{n}\right)$ the Banach space of continuous functions from $I$ to $\mathbb{R}^{n}$ with the norm $\|x\|_{\infty}=\sup \{|x|, x \in I\}$. We denote by $L^{1}\left(I, \mathbb{R}^{n}\right)$ the space of Lebesgue-integrable function $x: I \longrightarrow \mathbb{R}^{n}$ with the norm

$$
\|x\|_{L^{1}}=\int_{0}^{1}|x(t)| \mathrm{d} t
$$

Let

$$
X=\left\{\begin{array}{c}
x \in C\left(J, \mathbb{R}^{n}\right) \\
x^{\prime \prime} \in C\left(I ; \mathbb{R}^{n}\right)
\end{array}\right\}
$$

with the norm

$$
\|x\|_{X}=\|x\|_{\infty}+\left\|x^{\prime \prime}\right\|_{\infty}
$$

Definition 1. The Riemann-Liouville integral of order $\alpha>0$ for a continuous function $\varphi \in L^{1}((0,1], \mathbb{R})$ is given by

$$
I^{\alpha} \varphi(t)=\frac{1}{\Gamma(\alpha)} \int_{0}^{t}(t-\tau)^{\alpha-1} \varphi(\tau) \mathrm{d} \tau, \quad \forall t \in(0,1]
$$

with $\Gamma(\alpha)$ : $=\int_{0}^{\infty} e^{-u} u^{\alpha-1} \mathrm{~d} u$.

Definition 2. If $\varphi \in C^{n}([0,1], \mathbb{R})$ and $n-1<\alpha \leq n$, then the Caputo fractional derivative is given by

$$
D^{\alpha} \varphi(t)=I^{n-\alpha} \frac{\mathrm{d}^{n}}{\mathrm{~d} t^{n}}(\varphi(t))=\frac{1}{\Gamma(n-\alpha)} \int_{0}^{t}(t-s)^{n-\alpha-1} \varphi^{(n)}(s) \mathrm{d} s
$$

Lemma 1. Let $n \in \mathbb{N}^{*}$ and $n-1<\alpha<n$, then the general solution of $D^{\alpha} u(t)=0$ is given by

$$
u(t)=\sum_{i=0}^{n-1} c_{i} t^{i}
$$

such that $c_{i} \in \mathbb{R}, \quad i=0,1,2, \ldots, n-1$.

$$
I^{\alpha} D^{\alpha} u(t)=u(t)+\sum_{k=0}^{n-1} \frac{u^{(k)}(0)}{k !} t^{k}
$$

with $t>0, n-1<\alpha<n$.

Lemma 3. Let $1<\beta<2$ and $3<\alpha<4$. Then, it holds

Lemma 2. Taking $n \in \mathbb{N}^{*}$ and $n-1<\alpha<n$, we have

$$
I^{\alpha} D^{\beta} u(t)=I^{\alpha-\beta} u(t)-\frac{u(0) t^{\alpha-\beta}}{\Gamma(\alpha-\beta+1)}-\frac{u^{\prime}(0) t^{\alpha-\beta+1}}{\Gamma(\alpha-\beta+2)}
$$


Proof. For this proof, we use the same method in [28]. We have

$$
\begin{aligned}
I^{\alpha} D^{\beta} u(t) & =\frac{1}{\Gamma(\alpha) \Gamma(2-\beta)} \int_{0}^{t}(t-\tau)^{\alpha-1} \int_{0}^{t}(\tau-s)^{1-\beta} u^{\prime \prime}(s) \mathrm{d} s \mathrm{~d} \tau, \\
& =\frac{1}{\Gamma(\alpha) \Gamma(2-\beta)} \int_{0}^{t} u^{\prime \prime}(s) \mathrm{d} s \int_{s}^{t}(t-\tau)^{\alpha-1}(\tau-s)^{1-\beta} \mathrm{d} \tau .
\end{aligned}
$$

With the change of variable $\tau=s+(t-s) \eta$, we have

$$
\int_{s}^{t}(t-\tau)^{\alpha-1}(\tau-s)^{1-\beta} \mathrm{d} \tau=\frac{\Gamma(\alpha) \Gamma(2-\beta)}{\Gamma(\alpha-\beta+2)}(t-s)^{\alpha-\beta+1} .
$$

Now, we get

$$
I^{\alpha} D^{\beta} u(t)=\frac{1}{\Gamma(\alpha-\beta+2)} \int_{0}^{t}(t-s)^{\alpha-\beta+1} u^{\prime \prime}(s) \mathrm{d} s=I^{\alpha-\beta} u(t)-\frac{u(0) t^{\alpha-\beta}}{\Gamma(\alpha-\beta+1)}-\frac{u^{\prime}(0) t^{\alpha-\beta+1}}{\Gamma(\alpha-\beta+2)}
$$

Definition 3. Let $X$ be a Banach space. Then, a map $T: X \longrightarrow X$ is called a contraction mapping on $X$ if there exists $q \in[0,1)$ such that

$$
\|T(x)-T(y)\| \leq q\|x-y\|,
$$

for all $x, y \in X$.

Theorem 1 (Banach's fixed point theorem, see [29]). Let $\Omega$ be a nonempty closed subset of a Banach space $X$. Then, any contraction mapping $T$ of $\Omega$ into itself has a unique fixed point.

Theorem 2 (Schaefer's fixed point theorem, see [29]). Let $X$ be a Banach space, and let $N: X \longrightarrow X$ be a completely continuous operator. If the set
$E=\{y \in X: y=\lambda N y$ for some $\lambda \in(0,1)\}$ is bounded, then $N$ has fixed points.

\section{Main Results}

We begin this section by some results that help us for solving the problem considered in (1).

Lemma 4. For any $x \in X$ and $1<\beta<2$, we have

$$
\left\|D^{\beta} x\right\|_{\infty} \leq \frac{1}{\Gamma(3-\beta)}\left\|x^{\prime \prime}\right\|_{\infty} \leq \frac{1}{\Gamma(3-\beta)}\left\|x^{\prime \prime}\right\|_{X} .
$$

Proof. By the definition of the operator $D^{\beta}$, we have

$$
\left|D^{\beta} x(t)\right|_{\infty}=\frac{1}{\Gamma(2-\beta)}\left|\int_{0}^{t}(t-s)^{1-\beta} x^{\prime \prime}(s) \mathrm{d} s\right| \leq\left\|x^{\prime \prime}\right\|_{\infty} \frac{1}{\Gamma(2-\beta)} \int_{0}^{1}(1-s)^{1-\beta} \mathrm{d} s \leq \frac{1}{\Gamma(3-\beta)}\left\|x^{\prime \prime}\right\|_{\infty} .
$$


Lemma 5. Let $1<\beta<2,3<\alpha<4$, and $G \in C\left(I, \mathbb{R}^{n}\right)$. Then, has for solution the following function we can state that the problem,

$$
\left\{\begin{array}{l}
D^{\alpha} x(t)-A D^{\beta} x(t)=G(t), \quad t \in I=[0,1] \\
x(0)=x_{0} \\
x^{\prime}(0)=x_{0}^{\prime} \\
x^{\prime \prime}(0)=x_{0}^{\prime \prime} \\
x^{\prime \prime \prime}(0)=x_{0}^{\prime \prime}
\end{array}\right.
$$

$$
\begin{aligned}
x(t)= & x_{0}+x_{0}^{\prime} t+\frac{1}{2} x_{0}^{\prime \prime} t^{2}+\frac{1}{6} x_{0}^{\prime \prime} t^{3}-\frac{A t^{\alpha-\beta}}{\Gamma(\alpha-\beta+1)} x_{0}-\frac{A t^{\alpha-\beta+1}}{\Gamma(\alpha-\beta+2)} x_{0}^{\prime} \\
& +\frac{A}{\Gamma(\alpha-\beta)} \int_{0}^{t}(t-s)^{\alpha-\beta-1} x(s) \mathrm{d} s+\frac{1}{\Gamma(\alpha)} \int_{0}^{t}(t-s)^{\alpha-1} G(s) \mathrm{d} s .
\end{aligned}
$$

Proof. By applying $I^{\alpha}$ to both sides of equation (16), we have

$$
I^{\alpha} D^{\alpha} x(t)-A I^{\alpha} D^{\beta} x(t)=I^{\alpha} G(t),
$$

and using the property established in Lemmas 2 and 3, we find that

$$
\begin{aligned}
x(t)= & x(0)+x^{\prime}(0) t+\frac{1}{2} x^{\prime \prime}(0) t^{2}+\frac{1}{6} x^{\prime \prime \prime}(0) t^{3}-\frac{A t^{\alpha-\beta}}{\Gamma(\alpha-\beta+1)} x(0)-\frac{A t^{\alpha-\beta+1}}{\Gamma(\alpha-\beta+2)} x^{\prime}(0) \\
& +\frac{A}{\Gamma(\alpha-\beta)} \int_{0}^{t}(t-s)^{\alpha-\beta-1} x(s) \mathrm{d} s+\frac{1}{\Gamma(\alpha)} \int_{0}^{t}(t-s)^{\alpha-1} G(s) \mathrm{d} s .
\end{aligned}
$$

Some of the initial conditions allow us to have the result.

Conversely, assume that $x(t)$ satisfy the equation (16), then we see easily the initial conditions.

We use the fact $D^{\alpha} I^{\alpha} G(t)=G(t)$ and $D^{\alpha} C=0$, where $C$ is a constant; we get

$$
D^{\alpha} x(t)-A D^{\beta} x(t)=G(t), \quad t \in I=[0,1] .
$$

Let us now transform the above problem to a fixed point one. Consider the nonlinear operator $T: X \longrightarrow X$ defined by

$$
\begin{aligned}
T x(t)= & x_{0}+x_{0}^{\prime} t+\frac{1}{2} x_{0}^{\prime \prime} t^{2}+\frac{1}{6} x_{0}^{\prime \prime} t^{3}-\frac{A t^{\alpha-\beta}}{\Gamma(\alpha-\beta+1)} x_{0}-\frac{A t^{\alpha-\beta+1}}{\Gamma(\alpha-\beta+2)} x_{0}^{\prime} \\
& +\frac{A}{\Gamma(\alpha-\beta)} \int_{0}^{t}(t-s)^{\alpha-\beta-1} x(s) \mathrm{d} s+\frac{1}{\Gamma(\alpha)} \int_{0}^{t}(t-s)^{\alpha-1} f\left(t, x(t), D^{\beta} x(t), D^{\alpha} x(t)\right) \mathrm{d} s .
\end{aligned}
$$

To prove the main results, we need to work with the following hypotheses:
(H2) There exist nonnegative constants $c_{1}, c_{2}$, and $c_{3}<1$ such that, for any $t \in I, x_{1}, x_{2}, x_{3}, x_{1}^{*}, x_{2}^{*}, x_{3}^{*} \in \mathbb{R}^{n}$,

(H1) The function $f$ defined on $I \times \mathbb{R}^{3 n}$ is continuous.

$$
\left|f\left(t, x_{1}, x_{2}, x_{3}\right)-f\left(t, x_{1}^{*}, x_{2}^{*}, x_{3}^{*}\right)\right| \leq c_{1}\left|x_{1}-x_{1}^{*}\right|+c_{2}\left|x_{2}-x_{2}^{*}\right|+c_{3}\left|x_{3}-x_{3}^{*}\right| .
$$


Also, we consider the quantities

$$
\begin{aligned}
& D_{1}=\frac{\|A\|}{\Gamma(\alpha-\beta+1)}+\frac{c_{1} \Gamma(3-\beta)+c_{3}\|A\|+c_{2}}{\left(1-c_{3}\right) \Gamma(\alpha+1) \Gamma(3-\beta)}, \\
& D_{2}=\frac{\|A\|}{\Gamma(\alpha-\beta-1)}+\frac{c_{1} \Gamma(3-\beta)+c_{3}\|A\|+c_{2}}{\left(1-c_{3}\right) \Gamma(\alpha-1) \Gamma(3-\beta)} .
\end{aligned}
$$

The first main result deals with the existence of a unique solution for (1). It is based on the application of Banach fixed point theorem for contraction mappings.
Proof. It is sufficient for us to prove that $H$ is a contraction mapping.

Let $(x, y) \in X^{2}$. Then, we can write

Theorem 3. If the conditions (H1) and (H2) are satisfied and $D<1\left(D:=D_{1}+D_{2}\right)$, then problem (1) has a unique solution on $I$.

$$
|T x(t)-T y(t)| \leq \frac{\|A\|}{\Gamma(\alpha-\beta)} \int_{0}^{t}(t-s)^{\alpha-\beta-1}|x(s)-y(s)| \mathrm{d} s+\frac{1}{\Gamma(\alpha)} \int_{0}^{t}(s-t)^{\alpha-1}|g(s)-h(s)| \mathrm{d} s,
$$

where $g, h \in C\left(I, \mathbb{R}^{n}\right) \quad$ defined by $\quad$ From (H2) for each $t \in I$, we have

$g(t)=f\left(t, x(t), D^{\beta} x(t), g(t)+A D^{\beta} x(t)\right)$

and

$h(t)=f\left(t, y(t), D^{\beta} y(t), h(t)+A D^{\beta} y(t)\right)$.

$$
|g(t)-h(t)| \leq c_{1}|x(t)-y(t)|+c_{2}\left|D^{\beta}(x(t)-y(t))\right|+c_{3}|g(t)-h(t)|+c_{3}\|A\||x(t)-y(t)|,
$$

and using Lemma 4 , we have

Therefore, we have for each $t \in I$,

$$
|g(t)-h(t)| \leq \frac{c_{1} \Gamma(3-\beta)+c_{3}\|A\|+c_{2}}{\left(1-c_{3}\right) \Gamma(3-\beta)}\|x-y\|_{X} .
$$

$$
\|T x-T y\|_{\infty} \leq\left[\frac{\|A\|}{\Gamma(\alpha-\beta+1)}+\frac{c_{1} \Gamma(3-\beta)+c_{3}\|A\|+c_{2}}{\left(1-c_{3}\right) \Gamma(\alpha+1) \Gamma(3-\beta)}\right]\|x-y\|_{X} \leq D_{1}\|x-y\|_{X} .
$$

On the other hand, we have

$$
\begin{aligned}
(T x)^{\prime \prime}(t)= & x_{0}^{\prime \prime}+x_{0}^{\prime \prime} t-\frac{A t^{\alpha-\beta-2}}{\Gamma(\alpha-\beta-1)} x_{0}-\frac{A t^{\alpha-\beta-1}}{\Gamma(\alpha-\beta)} x_{0}^{\prime}+\frac{A}{\Gamma(\alpha-\beta-2)} \int_{0}^{t}(t-s)^{\alpha-\beta-3} x(s) \mathrm{d} s \\
& +\frac{1}{\Gamma(\alpha-2)} \int_{0}^{t}(t-s)^{\alpha-3} f\left(t, x(t), D^{\beta} x(t), D^{\alpha} x(t)\right) \mathrm{d} s,
\end{aligned}
$$


which is clear in $C\left(I, \mathbb{R}^{n}\right)$.

Then, with the same arguments as before, we have

$$
\left\|(T x)^{\prime \prime}-(T y)^{\prime \prime}\right\|_{\infty} \leq\left[\frac{\|A\|}{\Gamma(\alpha-\beta-1)}+\frac{c_{1} \Gamma(3-\beta)+c_{3}\|A\|+c_{2}}{\left(1-c_{3}\right) \Gamma(\alpha-1) \Gamma(3-\beta)}\right]\|x-y\|_{X} \leq D_{2}\|x-y\|_{X} .
$$

Thus, we have

$$
\|T x-T y\|_{X} \leq\left(D_{1}+D_{2}\right)\|x-y\|_{X} .
$$

Since $D<1$, then the operator $T$ is contraction. Hence, by Banach's contraction principle, $T$ has a unique fixed point which is the unique solution of problem (1).

The following main result deals with the existence of at least one solution of the studied problem.

Theorem 4. Under the hypotheses (H1) and (H2), problem (1) has at least one solution $u(t), t \in I$.
Proof. Let us prove the result by considering the following steps:

Continuous of $T$ : if the proof is trivial, then it is omitted (we just apply the fact that $f$ is continuous.

Uniform boundness of $T$ : let us take $r>0$ and consider the (bounded) ball $B_{r}:=\left\{x \in X ;\|x\|_{X} \leq r\right\}$. For $y \in B_{r}$, we can write

$$
|T y(t)| \leq\left|x_{0}\right|+\frac{1}{2}\left|x_{0}^{\prime \prime}\right|+\frac{1}{6}\left|x_{0}^{\prime \prime}\right|+\frac{\|A\|\left|x_{0}\right|}{\Gamma(\alpha-\beta+1)}+\frac{\|A\|\left|x_{0}^{\prime}\right|}{\Gamma(\alpha-\beta+2)}+\frac{\|A\|}{\Gamma(\alpha-\beta-1)}\|y\|_{\infty}+\frac{1}{\Gamma(\alpha+1)}\|g\|_{\infty} .
$$

With a simple calculus, we get

$$
\|g\|_{\infty} \leq \frac{c_{1} \Gamma(3-\beta)+c_{3}\|A\|+c_{2}}{\left(1-c_{3}\right) \Gamma(3-\beta)}\|y\|_{X}+m^{*}
$$

where $m^{*}=\sup _{t \in I}|f(t, 0,0,0)|$.

Then, we have

$$
\|T y\|_{\infty} \leq\left|x_{0}\right|+\frac{1}{2}\left|x_{0}^{\prime \prime}\right|+\frac{1}{6}\left|x_{0}^{\prime \prime}\right|+\frac{\|A\|\left|x_{0}\right|}{\Gamma(\alpha-\beta+1)}+\frac{\|A\|\left|x_{0}^{\prime}\right|}{\Gamma(\alpha-\beta+2)}+\frac{m^{*}}{\Gamma(\alpha+1)}+D_{1} r<+\infty,
$$

and also we have

$$
\left\|(T y)^{\prime \prime}\right\|_{\infty} \leq\left|x_{0}^{\prime \prime}\right|+\mid x_{0}^{\prime \prime}+\frac{\|A\|\left|x_{0}\right|}{\Gamma(\alpha-\beta-1)}+\frac{\|A\|\left|x_{0}^{\prime}\right|}{\Gamma(\alpha-\beta)}+\frac{m^{*}}{\Gamma(\alpha-1)}+D_{2} r<+\infty .
$$

The above two inequalities show that $\|T y\|_{X}<+\infty$.

Consequently, $T$ is uniformly bounded.

Equicontinuity of $T$ : we prove that, for any bounded set $B_{r}$ for instance, we obtain that $T\left(B_{r}\right)$ is an equicontinuous set of $X$.
Take $t_{1}, t_{2} \in[0,1], t_{1}<t_{2}$ and consider the above (bounded) ball $B_{r}$ of $X$. So, by considering $y \in B_{r}$, we can state that

$$
\begin{aligned}
\left|T y\left(t_{2}\right)-T y\left(t_{1}\right)\right| \leq & \left|x_{0}^{\prime}\right|\left|t_{2}-t_{1}\right|+\frac{1}{2}\left|x_{0}^{\prime \prime}\right|\left|t_{2}^{2}-t_{1}^{2}\right|+\frac{1}{6}\left|x_{0}^{\prime \prime}\right|\left|t_{2}^{3}-t_{1}^{3}\right|+\frac{\|A\|\left|x_{0}^{\prime}\right|}{\Gamma(\alpha-\beta+1)}\left|t_{2}^{\alpha-\beta}-t_{1}^{\alpha-\beta}\right| \\
& +\frac{\|A\|\left|x_{0}^{\prime \prime}\right|}{\Gamma(\alpha-\beta+2)}\left|t_{2}^{\alpha-\beta+1}-t_{1}^{\alpha-\beta+1}\right|+\frac{\|A\| r}{\Gamma(\alpha-\beta+1)}\left|t_{2}^{\alpha-\beta}-t_{1}^{\alpha-\beta}\right|+\frac{M}{\Gamma(\alpha+1)}\left|t_{2}^{\alpha}-t_{1}^{\alpha}\right|
\end{aligned}
$$


where

$\left./\left(1-c_{3}\right) \Gamma(3-\beta)\right) r+m^{*}$.

$$
M=\left(c_{1} \Gamma(3-\beta)+c_{3}\|A\|+c_{2}\right.
$$

As $t_{2} \longrightarrow t_{1}$, the right-hand side of the above inequality tends to zero, and we have also

$$
\begin{aligned}
\left|(T y)^{\prime \prime}\left(t_{2}\right)-(T y)^{\prime \prime}\left(t_{1}\right)\right| \leq & \left|x_{0}^{\prime \prime}\right|\left|t_{2}-t_{1}\right|+\frac{\|A\|\left|x_{0}^{\prime}\right|}{\Gamma(\alpha-\beta-1)}\left|t_{2}^{\alpha-\beta-2}-t_{1}^{\alpha-\beta-2}\right|+\frac{\|A\|\left|x_{0}^{\prime \prime}\right|}{\Gamma(\alpha-\beta)}\left|t_{2}^{\alpha-\beta-1}-t_{1}^{\alpha-\beta-1}\right| \\
& +\frac{\|A\| r}{\Gamma(\alpha-\beta-1)}\left|t_{2}^{\alpha-\beta-2}-t_{1}^{\alpha-\beta-2}\right|+\frac{M}{\Gamma(\alpha-1)}\left|t_{2}^{\alpha-2}-t_{1}^{\alpha-2}\right| .
\end{aligned}
$$

As $t_{2} \longrightarrow t_{1}$, the right-hand side of the above inequality tends to zero. From a consequence of the AscoliArzela's theorem, we conclude that $T$ is completely continuous.

Boundness of $A_{\gamma}$ : the set $A_{\gamma}:=\{x \in X: x=\gamma T x, \gamma \in] 0,1[\}$ is bounded.
Let $y \in A_{\gamma}$. Then, we have $y=\gamma T y$ for some $0<\gamma<1$. Hence, we can write

$$
\begin{aligned}
\|y\|_{\infty} & \leq \gamma\left(\left|x_{0}\right|+\frac{1}{2}\left|x_{0}^{\prime \prime}\right|+\frac{1}{6}\left|x_{0}^{\prime \prime}\right|+\frac{\|A\|\left|x_{0}\right|}{\Gamma(\alpha-\beta+1)}+\frac{\|A\|\left|x_{0}^{\prime}\right|}{\Gamma(\alpha-\beta+2)}+\frac{m^{*}}{\Gamma(\alpha+1)}+D_{1} r\right), \\
\left\|(y)^{\prime \prime}\right\|_{\infty} & \leq \gamma\left(\left|x_{0}^{\prime \prime}\right|+\left|x_{0}^{\prime \prime}\right|+\frac{\|A\|\left|x_{0}\right|}{\Gamma(\alpha-\beta-1)}+\frac{\|A\|\left|x_{0}^{\prime}\right|}{\Gamma(\alpha-\beta)}+\frac{m^{*}}{\Gamma(\alpha-1)}+D_{2} r\right) .
\end{aligned}
$$

From (37) and (38), we state that $\|y\|_{X}<\infty$. The set is thus bounded.

Consequently, thanks to Schaefer fixed point theorem, we deduce that $T$ has at least one fixed point. Thus, problem (1) has a solution.

$$
\left\{\begin{array}{l}
D^{\alpha} x(t)-A D^{\beta} x(t)=f\left(t, x(t), D^{\beta} x(t), D^{\alpha} x(t)\right), \quad t \in I=[0,1] \\
x(0)=\left(\frac{1}{2}, 0\right) \\
x^{\prime}(0)=\left(0, \frac{1}{2}\right) \\
x^{\prime \prime}(0)=\left(\frac{1}{2}, 0\right) \\
x^{\prime \prime \prime}(0)=\left(0, \frac{1}{2}\right)
\end{array}\right.
$$


where

$$
\begin{aligned}
& f:[0,1] \times \mathbb{R}^{2} \times \mathbb{R}^{2} \times \mathbb{R}^{2} \longrightarrow \mathbb{R}^{2}, \\
& (t, u, v, w) \mapsto\left(\frac{1}{10 e^{t+5}(1+\|u\|+\|v\|+\|w\|)}, \frac{1}{10 e^{t+7}(1+\|u\|+\|v\|+\|w\|)}\right),
\end{aligned}
$$

with $\|u\|=\max \left\{x_{1}, x_{2}\right\}, \quad u=\left(x_{1}, x_{2}\right)$. We take $\quad$ For any $u, v, w, \bar{u}, \bar{v}, \bar{w} \in \mathbb{R}^{2}$ and $t \in[0,1]$, $A=\left(\begin{array}{cc}(1 / 20) & 0 \\ 0 & 0\end{array}\right), \alpha=(15 / 4)$, and $\beta=(3 / 2)$.

We can see clearly that the function $f$ is continuous.

$$
\begin{aligned}
& \left|\frac{1}{10 e^{t+5}(1+\|\bar{u}\|+\|\bar{v}\|+\|\bar{w}\|)}-\frac{1}{10 e^{t+5}(1+\|\bar{u}\|+\|\bar{v}\|+\|\bar{w}\|)}\right| \leq \frac{1}{10 e^{5}}(|u-\bar{u}|+|v-\bar{v}|+|w-\bar{w}|), \\
& \left|\frac{1}{10 e^{t+7}(1+\|\bar{u}\|+\|\bar{v}\|+\|\bar{w}\|)}-\frac{1}{10 e^{t+7}(1+\|\bar{u}\|+\|\bar{v}\|+\|\bar{w}\|)}\right| \leq \frac{1}{10 e^{7}}(|u-\bar{u}|+|v-\bar{v}|+|w-\bar{w}|),
\end{aligned}
$$

which give

$$
|f(t, u, v, w)-f(t, \bar{u}, \bar{v}, \bar{w})| \leq c_{1}|u-\bar{u}|+c_{2}|v-\bar{v}|+c_{3}|w-\bar{w}|
$$

where $c_{1}=c_{2}=c_{3}=\left(1 / 10 e^{5}\right)$.

Hence, the hypotheses (H1) and (H2) are satisfied.

With a simple computation, we get $D_{1}=0,0807$ and $D_{2}=0,24$, which imply $D<1$.

Thus, all the assumptions from (H1)-(H3) are satisfied. From Theorem 3, we conclude that equation (1) has a unique solution.

\section{Conclusion}

In this work, we consider a nonlinear implicit fractional differential equation and we use the Caputo derivative operator. We prove two theorems and an example to illustrate our results. In the first theorem, we prove the existence and uniqueness of the solution and the second theorem deals with the existence of at least one solution. The methods used are the Banach's fixed point theorem and Schaefer's fixed point theorem. Here, two Caputo derivative operators of different fractional orders were used in the considered equation and it would be relevant to generalize this idea by considering several Caputo operators of different fractional orders.

\section{Data Availability}

No data were used to support this study.

\section{Conflicts of Interest}

The author declares no known conflicts of interest or personal relationships that could have appeared to influence the work reported in this paper.

\section{References}

[1] S. Abbas and M. Benchohra, "On the generalized Ulam-Hyers-Rassias stability for Darboux problem for partial fractional implicit differential equations," Applied Mathematics E-Notes, vol. 14, pp. 20-28, 2014.

[2] S. Abbas, M. Benchohra, J. Graef, and J. Henderson, "Implicit fractional differential and integral equations: existence and stability," in De Gruyter Series in Nonlinear Analysis and ApplicationsVol. 26, De Gruyter, Berlin, Germany, 2018.

[3] S. Abbas, M. Benchohra, and G. M. N'Guérékata, "Topics in fractional differential equations," in Developments in MathematicsVol. 27, Springer, New York, NY, USA, 2012.

[4] S. Abbas, M. Benchohra, and G. M. N'Guérékata, "Advanced fractional differential and integral equations," in Mathematics Research DevelopmentsNova Science Publishers, Inc., New York, NY, USA, 2015.

[5] M. A. Dokuyucu and H. Dutta, "A fractional order model for Ebola virus with the new Caputo fractional derivative without singular kernel," Chaos, Solitons \& Fractals, vol. 134, Article ID 109717, 2020. 
[6] M. A. Dokuyucu, "Caputo and atangana-baleanu-caputo fractional derivative applied to Garden equation," Turish Journal of Sciences, vol. 5, no. 1, pp. 1-7, 2020.

[7] A. A. Kilbas, H. M. Srivastava, and J. J. Trujillo, "Theory and applications of fractional differential equations," in NorthHolland Mathematics StudiesElsevier Science B.V., Amsterdam, Netherlands, 2006.

[8] K. S. Miller and B. Ross, An Introduction to the Fractional Calculus and Fractional Differential Equations, A WileyInterscience Publication. John Wiley and Sons, Inc., New York, NY, USA, 1993.

[9] N. Sene and A. Ndiaye, "Fractional model for a class of diffusion-reaction equation represented by the fractionalorder derivative," Fractal and Fractional, vol. 4, no. 2, 15 pages, Article ID 8815377, 2020.

[10] R. P. Agarwal, D. O'Regan, and S. Staněk, "Positive solutions for mixed problems of singular fractional differential equations," Mathematische Nachrichten, vol. 285, no. 1, 2012.

[11] Z. Bai and W. Sun, "Existence and multiplicity of positive solutions for singular fractional boundary value problems," Computers \& Mathematics with Applications, vol. 63, no. 9, 2012.

[12] Z. Bekkouche, Z. Dahmani, and G. Zhang, "Solutions and stabilities for a 2D-non homogeneous Lane-Emden fractional system," International Journal of Open Problems in Computer Science and Mathematics, vol. 11, no. 2, 2018.

[13] Y. Gouari, Z. Dahmani, and A. Ndiaye, "A generalized sequential problem of Lane Emden type via fractional calculus," Moroccan Journal of Pure and Applied Analysis, vol. 6, no. 2, Article ID 168183, 2020.

[14] R. W. Ibrahim, "Stability of a fractional differential equation," International Journal of Mathematical, Computational, Physical and Quantum Engineering, vol. 7, no. 3, 2013.

[15] M. Feckan, Y. Zhou, and J. Wang, "On the concept and existence of solution of an impulsive fractional differential equations," Communications in Nonlinear Science and $\mathrm{Nu}$ merical Simulation, vol. 17, p. 1627, 1996.

[16] T. L. Guo and W. Jiang, "Impulsive fractional functional differential equations," Computers \& Mathematics with Applications, vol. 64, no. 10, pp. 3414-3424, 2012.

[17] J. Wang, X. Li, and W. Wei, "On the natural solution of an impulsive fractional differential equation of order $q \in(1 ; 2)$," Communications in Nonlinear Science and Numerical Simulation, vol. 17, pp. 1381-1391, 2012.

[18] Y. Zhou, F. Jiao, and J. Li, "Existence and uniqueness for fractional neutral differential equations with infinite delay," Nonlinear Analysis: Theory, Methods \& Applications, vol. 71, no. 7-8, pp. 3249-3256, 2009.

[19] G. Chai, "Existence results for anti-periodic boundary value problems of fractional differential equations," Advances in Difference Equations, vol. 2013, no. 35, pp. 1-15, 2013.

[20] J. Sheng and W. Jiang, "Existence and uniqueness of the solution of fractional damped dynamical systems," Advances in Difference Equations, vol. 2017, no. 16, pp. 1-14, 2017.

[21] A. Keten, M. Yavuz, and D. Baleanu, "Nonlocal cauchy problem via a fractional operator involving power kernel in Banach spaces," Fractal and Fractional, vol. 3, no. 2, p. 27, 2019.

[22] M. A. Khan, Z. Hammouch, and D. Baleanu, "Modeling the dynamics of hepatitis E via the Caputo-Fabrizio derivative," Mathematical Modelling of Natural Phenomena, vol. 14, no. 3, p. 19, 2019.

[23] P. Veeresha, H. M. Baskonus, D. G. Prakasha, W. Gao, and G. Yel, "Regarding new numerical solution of fractional schistosomiasis disease arising in biological phenomena," Chaos Solitons Fractals, vol. 133, Article ID 109661, 2020.

[24] X.-J. Yang, M. Abdel-Aty, and C. Cattani, "A new general fractional-order derivataive with Rabotnov fractional-exponential kernel applied to model the anomalous heat transfer," Thermal Science, vol. 23, no. 3, pp. 1677-1681, 2019.

[25] M. Yavuz, "Characterizations of two different fractional operators without singular kernel," Mathematical Modelling of Natural Phenomena, vol. 14, no. 3, p. 13, 2019.

[26] A. Yokus and S. Gulbahar, "Numerical solutions with linearization techniques of the fractional Harry Dym equation," Applied Mathematics and Nonlinear Sciences, vol. 4, no. 1, pp. 35-41, 2019.

[27] S. Abbas, M. Benchohra, S. Bouriah, and J. Henderson, "Existence and uniqueness results for nonlinear implicit fractional systems," Journal of Nonlinear Evolution Equations and Applications, no. 5, pp. 81-94, 2019.

[28] X. L. Dind, "Controllability and optimality of linear timeinvariant neutral control systems with different fractional orders," Acta Mathematica Scientia, vol. 35B, no. 5, pp. 1003-1013, 2015.

[29] A. Granas and J. Dugundji, Fixed Point Theory, SpringerVerlag, New York, NY, USA, 2003. 